\title{
Infective Tricuspid Valve Endocarditis with Pulmonary Emboli Caused by Campylobacter Fetus after Tooth Extraction
}

\author{
Keisuke Miki, Ryoji Maekura, Toru Hiraga, Atsushi Hirotani, Hisako Hashimoto, \\ Seigo Kitada, Mari Miki, Kenji YoshimUra, Nobuyuki NAKA, Masaharu Motone, \\ Takeya FuJIKAWA, Shodayu TAKASHIMA*, Rika KITAZUME**, Hideaki KANZAKI**, \\ Satoshi NAKATANI**, Hirotaka WATANUKI***, Osamu TAGUSARI***, \\ Junjiro KoBAYASHI*** and Masami ITO
}

\begin{abstract}
We describe a case of infective endocarditis caused by Campylobacter fetus accompanied by pulmonary emboli. A 52-year-old man was referred to our hospital due to febrile temperatures with a history of dental treatment followed by eating raw meat. Computed tomography revealed multiple infiltrations and a nodule with low attenuation area and feeding vessels. A mobile mass, possible vegetation, attached to the tricuspid valve was detected by transthoracic echocardiography. Two blood cultures disclosed Campylobacter fetus. Long-term antibiotic therapy was given, curing the infection with valvuloplasty. We presented the possibility that infective Campylobacter fetus endocarditis after dental treatment was caused by eating raw meat.

(Internal Medicine 44: 1055-1059, 2005)
\end{abstract}

Key words: valvuloplasty, dental treatment

\section{Introduction}

Campylobacters are motile, non-spore-forming, curved gram-negative rods. To date, more than 15 different Campylobacter species have been identified. Campylobacter fetus, comprising two subspecies, C. fetus subsp. fetus (referred to as C. fetus) and C. fetus subsp. venerealis can cause disease in both humans and animals (1). The most common type of infection with Campylobacter species is diarrhea, with Campylobacter jejuni. In contrast, C. fetus has been recognized as a major species causing extraintestinal illnesses since the early 1900s (2). In about 20 cases of infective C. fetus endocarditis, up to $60 \%$ of the patients had underlying heart disease with valvular abnormalities and cardiomyopathy (3). In reported cases of infective C. fetus endocarditis, the aortic valve is usually involved, but rightsided endocarditis is uncommon (4). Right-sided endocarditis occurs in intravenous drug abusers and intensive care patients with peripheral or central venous catheters, most often involving the tricuspid valve (5). Although C. fetus can be transmitted through the ingestion of contaminated food or through animal exposure $(6,7)$, the route and source of $C$. fetus infection in humans remains to be clearly elucidated.

Herein, we outline the case of a patient, with neither an underlying disease nor a history of drug abuse, of infective C. fetus endocarditis who had undergone tooth extraction followed by eating raw meat as a stable food.

\section{Case report}

A 52-year-old man underwent dental treatments between March and May 2004, including a tooth extraction on May 8. He likes eating raw meat served in the Korean ('yukke' or 'namareba') style, and ate it several times during the period. Two weeks after his tooth extraction, he developed a chill and a fever of $38^{\circ} \mathrm{C}$. On June 3, he visited to another hospital because of high fever and abnormal chest X-ray results that were examined at mass screening on May 26 by chance. $\mathrm{He}$

From the Department of Internal Medicine and *the Department of Radiology, National Hospital Organization Toneyama National Hospital, Osaka, **the Department of Cardiology and ***the Cardiovascular Surgery, National Cardiovascular Center, Osaka

Received for publication December 24, 2004; Accepted for publication May 31, 2005

Reprint requests should be addressed to Dr. Keisuke Miki, the Department of Internal Medicine, National Hospital Organization Toneyama National Hospital, 5-1-1 Toneyama, Toyonaka, Osaka 560-8552 
had been treated with antibiotics (CPFX) as he was diagnosed as having pulmonary pneumoniae, and his fever diminished. However, he had a spiking fever again. Then, he was referred to our hospital for further examination, and was admitted on July 14, 2004. No medication had been taken prior to this illness. There were no abdominal symptoms. On admission, the physical examination revealed a blood pressure of $114 / 44 \mathrm{mmHg}$, a heart rate of 70 beats $/ \mathrm{min}$, and a body temperature of $40^{\circ} \mathrm{C}$. He had no obvious heart murmur. The following data was obtained: a WBC count of 14,410 $\mu / l$, and a differential cell count of $80.8 \%$ neutrophils, $11.5 \%$ lymphocytes, $4.8 \%$ monocytes, $0.8 \%$ eosinophils, and $0.5 \%$ basophils, an erythrocyte sedimentation (ESR) level of 70 $\mathrm{mm} /$ hour, a C-reactive protein (CRP) level of $11.5 \mathrm{mg} / \mathrm{dl}$, and an RF level of $264 \mathrm{IU} / \mathrm{ml}$. Tests for the human immunodeficiency virus (HIV), mycobacterium tuberculosis, and connective tissue disease revealed no underlying disease. Two blood cultures drawn 12 hours apart grew the same C. fetus. A chest X-ray showed a focal shadow in the middle field of the right lung and small nodules in the lower field of the left lung (Fig. 1). By chest computed-tomography (CT) and HRCT, the former lesion was revealed to be a subpleurally located nodule of $2 \mathrm{~cm}$ in size with feeding vessels and a necrotic center (Fig. 2). A transthoracic echocardiography showed a large vegetation of $1.3 \times 0.9 \mathrm{~cm}$ in size on the anterior leaflet of tricuspid valve (Fig. 3). The only other finding was a mild tricuspid regurgitation. From these findings, this case was diagnosed as having infective $\mathrm{C}$. fetus endocarditis accompanied by pulmonary emboli, consistent with the diagnostic criteria of Duke (8). After collecting blood for culture on admission, intravenous PAPM/BP, 1 $\mathrm{g} /$ day, was given for 21 days, and changed to CLDM, 1.2 $\mathrm{g} / \mathrm{day}$, for 6 days due to the patient's liver disorder, considering the possible result of drug sensitivity. Then the levels CRP, ESR and RF decreased, and three cultures after antibiotic treatment did not grow C. fetus. There also followed a gradual improvement in chest CT findings but not in the right subpleular located nodule. However, on the day 23 post-admission, a sudden chest pain, with patient's desaturation suspected as relapsed pulmonary emboli occurred, and the transthoracic echocardiography revealed the vegetation to have increased in size slightly (on the day 24 postadmission the vegetation size, $1.8 \times 0.8 \mathrm{~cm}$ ). Then, the patient was refered to National Cardiovascular Center for surgical therapy on August 9. His new pulmonary emboli was confirmed using pulmonary perfusion scanning with $99 \mathrm{~m}$ Technetium macroaggregated albumin in the right middle lobe, although there was no obvious new shadow in Chest CT. At this time, the surgical therapy had not been carried out, since no elevation of CRP was found (CRP level of 0.24 $\mathrm{mg} / \mathrm{dl}$ ). CLDM, $1.2 \mathrm{~g} / \mathrm{day}$, was given continuously for 3 weeks after his transfer, however, he had a relapsed spiking fever from September 6. Then, he underwent tricuspid valvuloplasty with vegetation excising; a $2.5 \times 1.2 \mathrm{~cm}$ localized vegetation adhered to the anterior leaflet of the tricuspid valve was found at operation. C. fetus was not cultivated

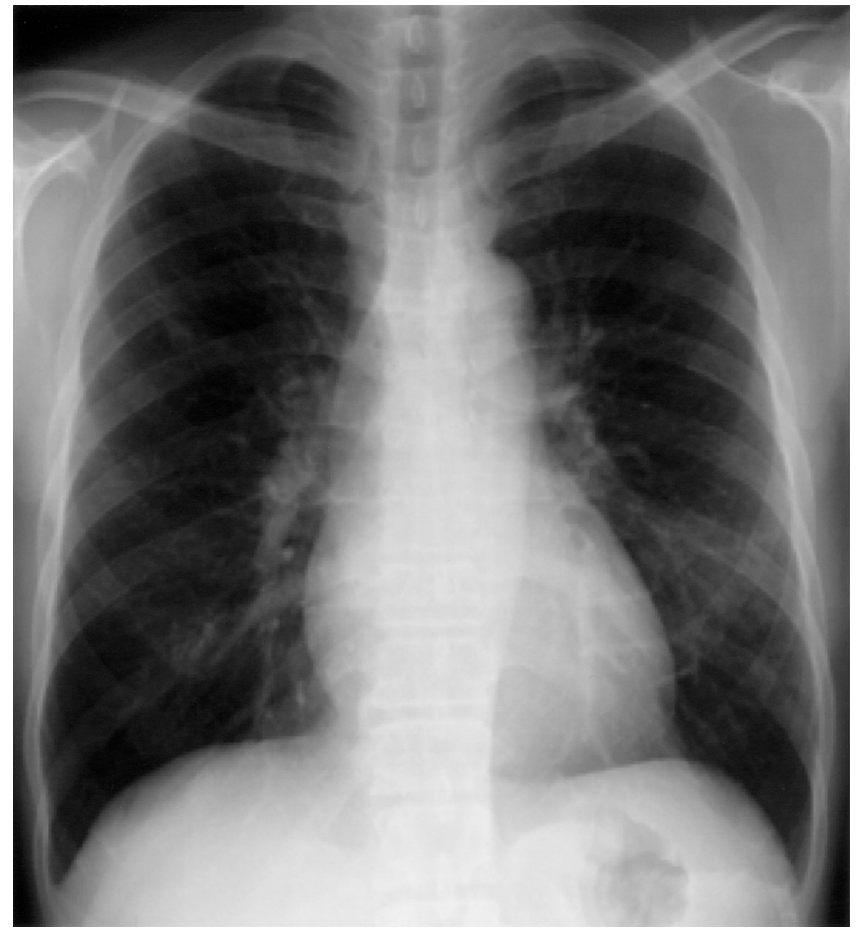

Figure 1. Chest X-ray revealed a focal opacity in the middle field of the right lung and small nodules in the lower field of the left lung.

from the vegetation. Pathological findings of the vegetation were compatible with infective endocarditis, in which inflammatory cells, such as neutrophils, were seen. Moreover, IPM/CS, $1.5 \mathrm{~g} / \mathrm{day}$, and GM, $120 \mathrm{mg} /$ day, were again given from September 6 for 6 weeks, and his condition has improved without relapsed infection.

\section{Discussion}

The tricuspid valve is affected in $5-10 \%$ of cases of infectious endocarditis, and the most common bacteria in tricuspid endocarditis is Staphylococcus auerus $(50-80 \%)(9,10)$. Infective endocarditis (IE) caused by C. fetus, especially without underlying disease, is uncommon (4). C. fetus infections in humans can result in serious systemic disease and even death, especially in compromised hosts $(1,4)$. However, the prevalence of $\mathrm{C}$. fetus infection may be underestimated because $\mathrm{C}$. fetus is difficult to isolate. It is important to know that $\mathrm{C}$. fetus grows at $25^{\circ} \mathrm{C}$ but not at $43^{\circ} \mathrm{C}$ in the laboratory, which differentiates it from the majority of other Campylobacter species (4). Also, we must maintain a high index of suspicion for this isolation in the clinical setting.

The pathogenesis of $\mathrm{C}$. fetus has been well defined of all Campylobactor infections, although it is uncommon. Early studies identified a loosely-attached capsular envelope, i. e. surface(S)-layer protein, from C. fetus which was later shown to render the organism's resistance to phagocytosis 

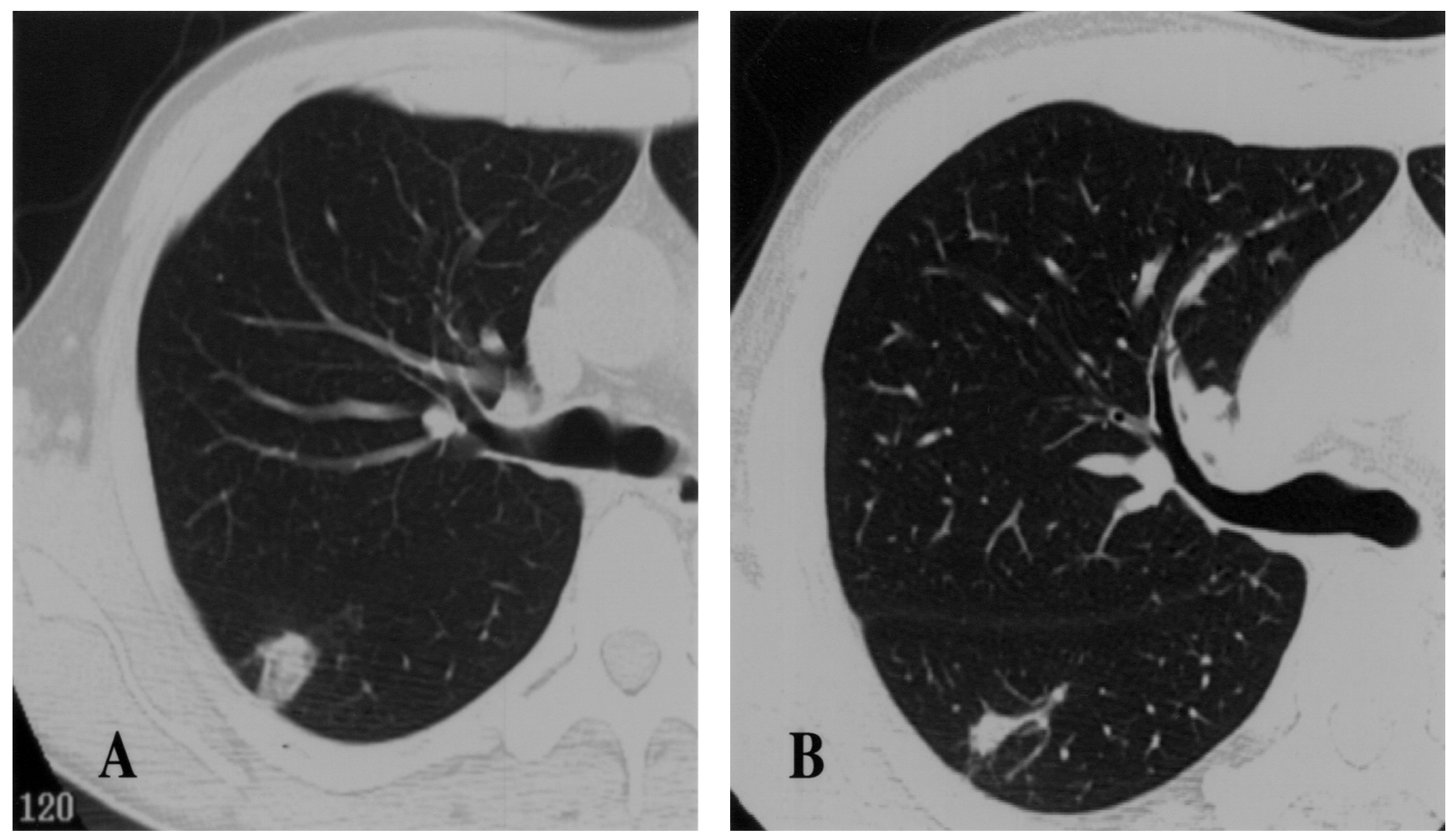

Figure 2. Chest CT (A) and HRCT (B) taken on admission showed a subpleural located lung nodule of $2 \mathrm{~cm}$ in size in the upper lobe (A) accompanied by feeding vessels (B).

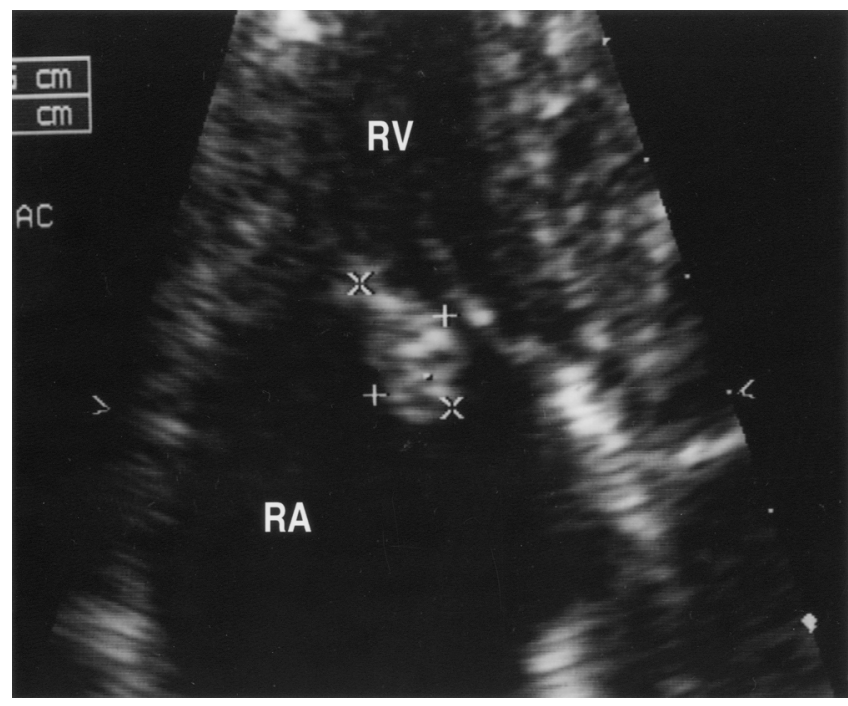

Figure 3. Transthoracic echocardiography on admission, showing a large vegetation of $1.3 \times 0.9 \mathrm{~cm}$ in size attached to the anterior leaflet of tricuspid valve. $\mathrm{RA}$ : right atrium, $\mathrm{RV}$ : right ventricle.

and complement-mediated killing (11). Moreover, the ability of the organism to switch surface-layer protein is believed to permit the organism's persistence in an immunologically hostile environment, especially in compromised hosts (12).

Direct contact with infected animals was reported as a possible mode of transmission. As another possible mode, C. fetus in humans can be transmitted through the ingestion of contaminated food or water, such as raw liver, raw meat, raw chicken, or raw milk $(6,7)$. More than $75 \%$ of $\mathrm{C}$. fetus infections occur in patients with debilitating disorders of the digestive tract (2). The organism has a tropism for damaged endovascular surfaces and can be present as a oral commensal, with dental disease predisposing to bacteremia, as in cases occurring after dental extraction. In one case, the mode of infection was thought to be the blood stream caused by eating raw chicken (13). This supports the same infection route occurring in the present patient, because he had no abdominal symptoms which might have caused the infection via the digestive tract in his clinical course, and oral contaminated food, i.e. raw meat, was the source of his infection.

Yet, our case report has some limitations. First, although we did not clarify the subspecies of C. fetus in our case, the possibility is high that our case had a C. fetus subspecies fetus infection. The reason is that $\mathrm{C}$. fetus subspecies venerealis may cause fertility problems in cows restrictedly, on the other hand, C. fetus subspecies fetus (C. fetus) is commonly recovered from the intestinal tract of many animal species and can cause serious systemic disease in humans (1). Second, an association between the organism and open 
tooth socket in our case was not found, since the sample from his dental pocket was not obtained due to it's localized improvement. Nevertheless, the route and source of our case are unique, while understandable predisposing factors and the infection route are generally detectable in infective endocarditis patents.

Kuhlman et al (14) reported the CT findings of septic pulmonary emboli. The most frequent pattern was multiple peripheral nodules of variable size. Other frequent findings included a feeding vessel sign, cavitation of nodules and wedge-shaped peripheral lesions abutting the pleura, and the densities extending into the pleural space. Moreover, chest CT first suggested the correct diagnosis of septic emboli, despite the absence of clinical suspicion. The characteristic CT findings of septic pulmonary emboli are useful for early and accurate diagnosis, and to treat adequately. In the present case, the distribution of lung lesions, their pattern, and multiplicity, although not common, all suggested hematogenous seeding via an open tooth socket from contaminated food as the source.

A variety of antibiotic treatments have been given as infectious endocarditis treatment, and it is necessary to keep in mind that tetracycline or chloramphenicol should be started empirically, but susceptibility testing should then be performed. Moreover, gentamicin, erythromycin, and imipenem are bactericidal by studies in vitro, although it is thought to a lesser extent than ampicillin (4). Because there is a high risk of relapse after short-term antibiotic therapy, prolonged therapy is recommended (a minimum 4 weeks of intravenous therapy) (13). However, occasionally the initial focus of infection might remain elusive, while the identity is generally clinically apparent. So, it is difficult to define the first choice of antibiotic drug and the appropriate duration of the therapy. Usually, infectious tricuspid endocarditis is well tolerated. This most likely reflects the benign nature of tricuspid valve involvement, compared to left side disease. Some investigators have suggested that recurrent pulmonary emboli and severe right-sided heart failure are predictors of a poor outcome in right-sided endcarditis $(15,16)$. In contrast, Hecht et al (10) reported a mortality rate of $7 \%$ and that the above factors were not associated with an adverse outcome, and should not be considered an indication for surgical therapy. They recommended that surgical therapy should not usually be warranted, except in cases of persistent or recurrent infection despite optimal antibiotic treatment. They also stated that vegetation size may be an important predictor of the outcome and that vegetation greater than 2.0 $\mathrm{cm}$ increases mortality. There is disagreement among authors concerning the surgical therapy on right-sided endocarditis. Moreover, the decision of procedure for tricuspid valve surgery is a difficult problem. Generally, tricuspid valvuloplasty is indicated for moderate to severe tricuspid insufficiency or tricuspid localized abnormalities. On the other hand, tricuspid valve replacement is performed for patients with significant organic tricuspid insufficiency that is unlikely to improve with another conservative therapy. In tricuspid valve replacement therapy, the choice of prosthesis remains controversial. In patients with mechanical prostheses, lifelong warfarin therapy is required. The lower pressure and flows in the right side of the heart highly predispose mechanical prostheses to valve thrombosis. In contrast, the degeneration of biological valves in the tricuspid position may be a problem due to calcification in late dysfunction. In cases such as the present case, valvuloplasty with vegetation excising should be performed whenever possible, since 1) the localized vegetation adhered to the anterior leaflet of tricuspid valve increased in size gradually and caused relapsed pulmonary emboli, and in particular relapsed infection occurred after long-term antibiotic therapy, 2) there is a risk of the above postoperative complications in valve replacement therapy, 3) the patient's age should be taken into account.

In conclusion, we described a patient with infective $\mathrm{C}$. fetus endocarditis accompanied by pulmonary emboli, who had undergone a tooth extraction, with a history of eating contaminated food. As such, C. fetus should be taken into account as one of the causative agents in patients with unexplained endocarditis, and this organism should be screened more frequently in such cases.

\section{References}

1) Wagenaar JA, van Bergen MA, Newell DG, Grogono-Thomas R, Duim B. Comparative study using amplified fragment length polymorphism fingerprinting, PCR genotyping, and phenotyping to differentiate Campylobacter fetus strains isolated from animals. J Clin Microbiol 39: 2283-2286, 2001.

2) Morrison VA, Lloyd BK, Chia JK, Tuazon CU. Cardiovascular and bacteremic manifestations of Campylobacter fetus infection: case report and review. Rev Infect Dis 12: 387-392, 1990.

3) Dzau VJ, Schur PH, Weinstein L. Vibrio fetus endocarditis in a patient with systemic lupus erythematosus. Am J Med Sci 272: 331-334, 1976.

4) Farrugia DC, Eykyn SJ, Smyth EG. Campylobacter fetus endocarditis: two case reports and review. Clin Infect Dis 18: 443-446, 1994.

5) Robbins MJ, Soeiro R, Frishman WH, Strom JA. Right-sided valvular endocarditis: etiology, diagnosis, and an approach to therapy. Am Heart J 111: 128-135, 1986.

6) Butzler JP, Dekeyser P, Lafontaine T. Susceptibility of related vibrios and Vibrio fetus to twelve antibiotics. Antimicrob Agents Chemother 5: 86-89, 1974.

7) Ward BQ. The apparent involvement of Vibrio fetus in an infection of man. J Bacteriol 55: 113-114, 1948.

8) Bayer AS, Ward JI, Ginzton LE, Shapiro SM. Evaluation of new clinical criteria for the diagnosis of infective endocarditis. Am J Med 96: 211-219, 1994.

9) Chan P, Ogilby JD, Segal B. Tricuspid valve endocarditis. Am Heart J 117: 1140-1146, 1989.

10) Hecht SR, Berger M. Right-sided endocarditis in intravenous drug users. Prognostic features in 102 episodes. Ann Intern Med 117: 560566, 1992.

11) Blaser MJ, Smith PF, Repine JE, Joiner KA. Pathogenesis of Campylobacter fetus infections. Failure of encapsulated Campylobacter fetus to bind C3b explains serum and phagocytosis resistance. J Clin Invest 81: 1434-1444, 1988.

12) Blaser MJ, Pei Z. Pathogenesis of Campylobacter fetus infections: critical role of high-molecular-weight S-layer proteins in virulence. J Infect Dis 167: 372-377, 1993.

13) Abe K, Nakamura $S$, Ninomiya $T$, et al. Infective endocarditis caused by Campylobacter fetus after allogeneic tooth transplantation: a case 


\section{Infective C. Fetus Endocarditis}

report. Br J Oral Maxillofac Surg 34: 230-234, 1996.

14) Kuhlman JE, Fishman EK, Teigen C. Pulmonary septic emboli: diagnosis with CT. Radiology 174: 211-213, 1990.

15) Barbour DJ, Roberts WC. Valve excision only versus valve excision plus replacement for active infective endocarditis involving the tricus- pid valve. Am J Cardiol 57: 475-478, 1986.

16) Yee ES, Ullyot DJ. Reparative approach for right-sided endocarditis. Operative considerations and results of valvuloplasty. J Thorac Cardiovasc Surg 96: 133-140, 1988. 